\title{
AN UPPER BOUND FOR THE MOMENTS OF A G.C.D. RELATED TO LUCAS SEQUENCES
}

\author{
DANIELE MASTROSTEFANO
}

\begin{abstract}
Let $\left(u_{n}\right)_{n \geq 0}$ be a non-degenerate Lucas sequence, given by the relation $u_{n}=$ $a_{1} u_{n-1}+a_{2} u_{n-2}$. Let $\ell_{u}(m)=l c m\left(m, z_{u}(m)\right)$, for $\left(m, a_{2}\right)=1$, where $z_{u}(m)$ is the rank of appearance of $m$ in $u_{n}$. We prove that

$$
\sum_{\substack{m>x \\\left(m, a_{2}\right)=1}} \frac{1}{\ell_{u}(m)} \leq \exp (-(1 / \sqrt{6}-\varepsilon+o(1)) \sqrt{(\log x)(\log \log x)}),
$$

when $x$ is sufficiently large in terms of $\varepsilon$, and where the $o(1)$ depends on $u$. Moreover, if $g_{u}(n)=\operatorname{gcd}\left(n, u_{n}\right)$, we will show that for every $k \geq 1$,

$$
\sum_{n \leq x} g_{u}(n)^{k} \leq x^{k+1} \exp (-(1+o(1)) \sqrt{(\log x)(\log \log x)}),
$$

when $x$ is sufficiently large and where the $o(1)$ depends on $u$ and $k$. This gives a partial answer to a question posed by C. Sanna. As a by-product, we derive bounds on $\#\left\{n \leq x:\left(n, u_{n}\right)>y\right\}$, at least in certain ranges of $y$, which strengthens what already obtained by Sanna. Finally, we start the study of the multiplicative analogous of $\ell_{u}(m)$, finding interesting results.
\end{abstract}

\section{INTRODUCTION}

Let $\left(u_{n}\right)_{n \geq 0}$ be an integral linear recurrence, that is, $\left(u_{n}\right)_{n \geq 0}$ is a sequence of integers and there exist $a_{1}, \ldots, a_{k} \in \mathbb{Z}$, with $a_{k} \neq 0$, such that

$$
u_{n}=a_{1} u_{n-1}+\cdots+a_{k} u_{n-k},
$$

for all integers $n \geq k$, with $k$ a fixed positive integer. We recall that $\left(u_{n}\right)_{n \geq 0}$ is said to be non-degenerate if none of the ratios $\alpha_{i} / \alpha_{j}(i \neq j)$ is a root of unity, where $\alpha_{1}, \ldots, \alpha_{r} \in \mathbb{C}$ are all the pairwise distinct roots of the characteristic polynomial

$$
f_{u}(X)=X^{k}-a_{1} X^{k-1}-\cdots-a_{k} .
$$

Moreover, $\left(u_{n}\right)_{n \geq 0}$ is said to be a Lucas sequence if $u_{0}=0, u_{1}=1$, and $k=2$. We note that the Lucas sequence with $a_{1}=a_{2}=1$ is known as the Fibonacci sequence. We refer the reader to [5, Chapter 1] for the basic terminology and theory of linear recurrences.

The function $g_{u}(n):=\operatorname{gcd}\left(n, u_{n}\right)$ has attracted the interest of several authors. For example, the set of fixed points of $g_{u}(n)$, or equivalently the set of positive numbers $n$ such that $n \mid u_{n}$, has been studied by Alba González, Luca, Pomerance, and Shparlinski [1], under the mild hypotheses that $\left(u_{n}\right)_{n \geq 0}$ is non-degenerate and that its characteristic polynomial has only simple roots. Moreover, this problem has been studied also by André-Jeannin [2], Luca and Tron [8], Sanna [11], Smyth [14] and Somer [15], when $\left(u_{n}\right)_{n \geq 0}$ is a Lucas or the Fibonacci sequence.

On the other hand, Sanna and Tron [12,13] have analysed the fiber $g_{u}(y)^{-1}$, when $\left(u_{n}\right)_{n \geq 0}$ is non-degenerate and $y=1$, and when $\left(u_{n}\right)_{n>0}$ is the Fibonacci sequence and $y$ is an arbitrary positive integer. Moreover, the image $g_{u}(\mathbb{N})$ has been investigated by Leonetti and Sanna [7], again when $\left(u_{n}\right)_{n \geq 0}$ is the Fibonacci sequence.

Other important questions about the function $g_{u}(n)$ are related to its behaviour on average and its distribution as arithmetic function. From now on, we focus on the specific case in which $\left(u_{n}\right)_{n \geq 0}$ is a non-degenerate Lucas sequence with non-zero discriminant $\Delta_{u}=a_{1}^{2}+4 a_{2}$. Otherwise, the sequence reduces to $u_{n}=n \alpha^{n}$, for a suitable $\alpha \in \mathbb{Z}$, and $g_{u}(n)=n$, for every positive integer $n$. Even in this particular situation, it is very difficult to find information on

1991 Mathematics Subject Classification. 11B39 11B37 (Primary) 11A05 11N64 (Secondary).

Key words and phrases. G.C.D. function; Lucas sequences; moments of arithmetic functions.

The author is funded by a Departmental Award and by an EPSRC Doctoral Training Partnership Award. 
the distribution of $g_{u}(n)$, because of its oscillatory behaviour. For this reason, it is natural to consider the flatter function $\log \left(g_{u}(n)\right)$, for which an asymptotic formula for its mean value, and more in general for its moments, has been given by Sanna, who proved the following theorem [10, Theorem 1.1].

Theorem 1.1. Fix a positive integer $\lambda$ and some $\varepsilon>0$. Then, for all sufficiently large $x$, how large depending on $a_{1}, a_{2}, \lambda$ and $\varepsilon$, we have

$$
\sum_{n \leq x}\left(\log g_{u}(n)\right)^{\lambda}=M_{u, \lambda} x+E_{u, \lambda}(x),
$$

where $M_{u, \lambda}>0$ is a constant depending on $a_{1}, a_{2}$ and $\lambda$, and the error term is bounded by

$$
E_{u, \lambda}(x) \ll_{u, \lambda} x^{(1+3 \lambda) /(2+3 \lambda)+\varepsilon} .
$$

Also, Sanna showed that the constant $M_{u, \lambda}$ can be expressed through a convergent series.

An immediate consequence of the previous result is the possibility of finding information about the distribution of $g_{u}$ [10, Corollary 1.3].

Corollary 1.2. For each positive integer $\lambda$, we have

$$
\#\left\{n \leq x: g_{u}(n)>y\right\} \ll_{u, \lambda} \frac{x}{(\log y)^{\lambda}},
$$

for all $x, y>1$.

In the same article, Sanna raised the question of finding an asymptotic formula for the moments of the function $g_{u}(n)$ itself. We are not able to answer to this apparently difficult question, but we can at least give a non-trivial estimate for them. The result is the following.

Theorem 1.3. For every integer $k \geq 1$ and $u_{n}$ a non-degenerate Lucas sequence, we have

$$
\sum_{n \leq x} g_{u}(n)^{k} \leq x^{k+1} \exp (-(1+o(1)) \sqrt{(\log x)(\log \log x)})
$$

as $x$ tends to infinity and where the $o(1)$ depends on $u$ and $k$.

For each positive integer $m$ relatively prime with $a_{2}$, let $z_{u}(m)$ be the rank of appearance of $m$ in the Lucas sequence $\left(u_{n}\right)_{n>0}$, that is, $z_{u}(m)$ is the smallest positive integer $n$ such that $m$ divides $u_{n}$. It is well known that $z_{u}(m)$ exists (see, e.g., [9]). Also, put $\ell_{u}(m):=l c m\left(m, z_{u}(m)\right)$. There is a simple trick to relate the moments of $g_{u}(n)$ with the rate of convergence of the series $\sum_{m>x,\left(m, a_{2}\right)=1} 1 / \ell_{u}(m)$, which has been partially studied by several authors. We will deduce a slightly weaker version of Theorem 1.3, in which the constant in the exponential is replaced by $-1 / \sqrt{6}+\varepsilon+o(1)$, for every $\varepsilon>0$, from it and the following bound.

Proposition 1.4. For every non-degenerate Lucas sequence $u_{n}$, we have

$$
\sum_{\substack{m>x \\\left(m, a_{2}\right)=1}} \frac{1}{\ell_{u}(m)} \leq \exp (-(1 / \sqrt{6}-\varepsilon+o(1)) \sqrt{(\log x)(\log \log x)}),
$$

when $x$ is large in terms of $\varepsilon$ and where the o(1) depends on $u$.

In the proof of Proposition 1.4 we highlight a method, based essentially on the distribution of smooth numbers, to achieve the above bound. It seems reasonable to think that a deeper analysis of the structure of $\ell_{u}(n)$ could lead to understand better the behaviour of $\sum_{m>x,\left(m, a_{2}\right)=1} 1 / \ell_{u}(m)$ and consequently to improve the result about the moments of $g_{u}(n)$. Nevertheless, using a completely different and more direct approach that we will describe later, we can obtain the stronger stated estimate in Theorem 1.3.

It is immediate to deduce from Theorem 1.3 the following improvement on the distribution of $g_{u}(n)$ at least when $y$ varies uniformly in a certain range. 
Corollary 1.5. We have

$$
\#\left\{n \leq x: g_{u}(n)>y\right\} \leq \frac{x^{2}}{y \exp \left(\left(1+o_{u}(1)\right) \sqrt{(\log x)(\log \log x)}\right)},
$$

for every $y \geq 1$, when $x$ is sufficiently large.

Proof. By using (1.5) with $k=1$ we obtain

$$
\begin{gathered}
\#\left\{n \leq x: g_{u}(n)>y\right\} \leq \sum_{n \leq x} \frac{g_{u}(n)}{y} \\
\leq \frac{x^{2}}{y \exp \left(\left(1+o_{u}(1)\right) \sqrt{(\log x)(\log \log x)}\right)}
\end{gathered}
$$

for every $y \geq 1$.

We observe that this is an improvement of (1.2), only for certain values of $y$, e.g. like for those satisfying

$$
x \exp \left(-\left(1 / 2+o_{u}(1)\right) \sqrt{(\log x)(\log \log x)}\right) \leq y \leq x .
$$

Consider now the multiplicative function $L_{u}(n)$ such that $L_{u}\left(p^{k}\right)=\ell_{u}\left(p^{k}\right)$, for every prime number $p \nmid a_{2}$ and power $k \geq 1$, and $L_{u}\left(p^{k}\right)=p^{k}$, otherwise. Using arguments coming from the theory of Dirichlet series of multiplicative functions, we end up with the following estimate.

Proposition 1.6. For every $u_{n}$ non-degenerate Lucas sequence, we have

$$
\sum_{n>x} \frac{1}{L_{u}(n)} \ll_{u} x^{-1 / 3+\varepsilon},
$$

for every $\varepsilon>0$, when $x$ is sufficiently large with respect to $\varepsilon$.

The above result shows that the lack of multiplicativity of $\ell_{u}(n)$ is the principle cause for the weaker upper bound in (1.4).

\section{Notations}

For a couple of real functions $f(x), g(x)$, with $g(x)>0$, we indicate with $f(x)=O(g(x))$ or $f(x) \ll g(x)$ that there exists an absolute constant $c>0$ such that $|f(x)| \leq c g(x)$, for $x$ sufficiently large. When the implicit constant $c$ depends from a parameter $\alpha$ we indicate the above bound with $f(x) \ll_{\alpha} g(x)$ or equivalently with $f(x)=O_{\alpha}(g(x))$.

Throughout, the letter $p$ is reserved for a prime number. We write $(a, b)$ and $[a, b]$ to denote the greatest common divisor and the least common multiple of integers $a, b$. As usual, we denote with $\lfloor w\rfloor$ the integer part of a real number $w$ and we indicate with $P(n)$ the greatest prime factor of a positive integer $n$.

\section{Preliminaries}

We begin by recalling the definition of the Jordan's totient function.

Definition 3.1. The Jordan's totient function of degree $k$ is defined as

$$
J_{k}(n)=n^{k} \prod_{p \mid n}\left(1-\frac{1}{p^{k}}\right),
$$

for every $k \geq 1$ and natural integers $n$.

Clearly, $J_{1}(n)=\varphi(n)$, the Euler's totient function, and it is immediate to see that $J_{k}(n)$ verifies the following identity.

Lemma 3.1. We have

$$
n^{k}=\sum_{d \mid n} J_{k}(d)
$$

for any $k \geq 1$ and natural integers $n$. 
The next lemma summarizes some basic properties of $\ell_{u}(n)$ and $z_{u}(n)$, which we will implicitly use later without further mention.

Lemma 3.2. For all positive integers $m, n$ and all odd prime numbers $p$, we have:

(1) $m \mid u_{n}$ if and only if $z_{u}(m) \mid n$ and $\left(m, a_{2}\right)=1$.

(2) $z_{u}([m, n])=\left[z_{u}(m), z_{u}(n)\right]$, whenever $\left(m n, a_{2}\right)=1$.

(3) $m \mid \operatorname{gcd}\left(n, u_{n}\right)$ if and only if $\left(m, a_{2}\right)=1$ and $\ell_{u}(m) \mid n$.

(4) $\ell_{u}([m, n])=\left[\ell_{u}(m), \ell_{u}(n)\right]$, whenever $\left(m n, a_{2}\right)=1$.

(5) $\ell_{u}\left(p^{j}\right)=p^{j} z_{u}(p)$ if $p \nmid \Delta_{u}$, and $\ell_{u}\left(p^{j}\right)=p^{j}$ if $p \mid \Delta_{u}$, for every $p \nmid a_{2}$ and $j \geq 1$.

(6) $z_{u}(p) \mid p \pm 1$, if $p \nmid \Delta_{u}$, and $z_{u}(p)=p$ if $p \mid \Delta_{u}$, for every $p \nmid a_{2}$.

For any $\gamma>0$, let us define

$$
\mathcal{Q}_{u, \gamma}:=\left\{p: p \nmid a_{2}, z_{u}(p) \leq p^{\gamma}\right\} .
$$

The following is [1, Lemma 2.1].

Lemma 3.3. For all $x^{\gamma}, y \geq 2$ and for any non-degenerate Lucas sequence $\left(u_{n}\right)_{n \geq 0}$, we have

$$
\#\left\{p: z_{u}(p) \leq y\right\} \ll_{u} \frac{y^{2}}{\log y}, \quad \mathcal{Q}_{u, \gamma}(x) \ll_{u} \frac{x^{2 \gamma}}{\gamma \log x} .
$$

It has been proven by Sanna and Tron [13, Lemma 3.2] that the series $\sum_{\left(n, a_{2}\right)=1} 1 / \ell_{u}(n)$ converges. We consider the following identity

$$
\sum_{\substack{n>x \\\left(n, a_{2}\right)=1}} \frac{1}{\ell_{u}(n)}=\sum_{\substack{n>x \\ P(n)>y \\\left(n, a_{2}\right)=1}} \frac{1}{\ell_{u}(n)}+\sum_{\substack{n>x \\ P(n) \leq y \\\left(n, a_{2}\right)=1}} \frac{1}{\ell_{u}(n)} .
$$

We note that the first sum in the right hand side of (3.2) has been already investigated by Sanna [10, Lemma 2.5] and we report here the result which he obtained.

Proposition 3.4. We have

$$
\sum_{\substack{\left(m, a_{2}\right)=1 \\ P(m)>y}} \frac{1}{\ell_{u}(m)} \ll_{u} \frac{1}{y^{1 / 3-\varepsilon}}
$$

for all $\varepsilon \in(0,1 / 4]$ and $y \gg_{u, \varepsilon} 1$.

Regarding the second sum in the right hand side of (3.2) we provide an estimate in the next lemma.

Lemma 3.5. Supposing that $y>(\log x)^{2}$ and $v=\log x / \log y$ tends to infinity as $x$ tends to infinity, we have

$$
\sum_{\substack{n>x \\ P(n) \leq y \\\left(n, a_{2}\right)=1}} \frac{1}{\ell_{u}(n)} \ll_{u}(\log y) e^{-\sqrt{y} / 2 \log y}+\frac{\log y}{\log v} e^{-v \log v} .
$$

Proof. Since $\ell_{u}(n) \geq n$, we may write

$$
\sum_{\substack{n>x \\ P(n) \leq y \\\left(n, a_{2}\right)=1}} \frac{1}{\ell_{u}(n)} \leq \int_{x}^{\infty} \frac{d \psi(t, y)}{t}
$$

where $\psi(t, y)$ is the counting function of the $y$-smooth numbers less than $t$. Clearly, we have

$$
\int_{x}^{\infty} \frac{d \psi(t, y)}{t}=\left.\frac{\psi(t, y)}{t}\right|_{x} ^{\infty}+\int_{x}^{\infty} \frac{\psi(t, y)}{t^{2}} d t
$$

To estimate the second term on the right hand side of (3.5) we suppose first that $y>\log ^{2}(x)$ and then we split it into two parts:

$$
\int_{x}^{\infty} \frac{\psi(t, y)}{t^{2}} d t=\int_{x}^{z} \frac{\psi(t, y)}{t^{2}} d t+\int_{z}^{\infty} \frac{\psi(t, y)}{t^{2}} d t
$$


where we put $z=e^{\sqrt{y}}$. Using the estimate [16, Theorem 1, §5.1, Chapter III]

$$
\psi(t, y) \ll t e^{-\log t / 2 \log y}=t^{1-1 / 2 \log y},
$$

valid uniformly for $t \geq y \geq 2$, we obtain

$$
\int_{z}^{\infty} \frac{\psi(t, y)}{t^{2}} \ll \int_{z}^{\infty} t^{-1-1 /(2 \log y)} d t \ll(\log y) z^{-1 /(2 \log y)}=(\log y) \exp \left(-\frac{\sqrt{y}}{2 \log y}\right) .
$$

By the Corollary of the Theorem 3.1 in [4], we know that

$$
\psi(t, y) \leq t \exp \left(-(1+o(1)) \frac{\log t}{\log y} \log \left(\frac{\log t}{\log y}\right)\right),
$$

in the region $y>\log ^{2} t$. Here the $o(1)$ is with respect to $\log t / \log y \rightarrow \infty$. If $v=\log x / \log y$ tends to infinity as $x$ tends to infinity, then we may use the simpler

$$
\psi(t, y) \leq t \exp \left(-\frac{\log t}{\log y} \log \left(\frac{\log t}{\log y}\right)\right),
$$

for any $x \leq t \leq z$. Note that equation (3.8) also follows from the aformentioned Corollary in [4]. Let us suppose to be in this situation. Now, inserting this bound and using the change of variable $s=\log t$, we get

$$
\int_{x}^{z} \frac{\psi(t, y)}{t^{2}} d t \leq \int_{\log x}^{\sqrt{y}} \exp \left(-\frac{s}{\log y} \log \left(\frac{s}{\log y}\right)\right) d s,
$$

which after another change of variable $s=w \log y$ becomes

$$
(\log y) \int_{\log x / \log y}^{\sqrt{y} / \log y} \exp (-w \log w) d w .
$$

Using that $w \geq v$ and putting $w \log v=r$, we find

$$
\int_{x}^{z} \frac{\psi(t, y)}{t^{2}} d t \leq \frac{\log y}{\log v} \int_{v \log v}^{\sqrt{y} \log v / \log y} e^{-r} d r \leq \frac{\log y}{\log v} e^{-v \log v}
$$

Regarding the first term on the right hand side of (3.5), we note that

$$
\left.\frac{\psi(t, y)}{t}\right|_{x} ^{\infty} \leq \lim _{t \rightarrow \infty} \frac{\psi(t, y)}{t} \ll \lim _{t \rightarrow \infty} t^{-1 / 2 \log y}=0
$$

by (3.6). Collecting the results, we obtain the estimate (3.4).

Finally, we can deduce the stated estimate on $\sum_{n>x} 1 / \ell_{u}(n)$.

Proof of Proposition 1.4. By Proposition 3.4 and Lemma 3.5 we conclude that

$$
\sum_{\substack{n>x \\\left(n, a_{2}\right)=1}} \frac{1}{\ell_{u}(n)} \ll_{u} \frac{1}{y^{1 / 3-\varepsilon}}+\frac{\log y}{\log v} e^{-v \log v},
$$

for every $\varepsilon>0$, if $y$ is sufficiently large in terms of $\varepsilon$. It is immediate to see that the best choice for $y$ is of the form $y=\exp (C \sqrt{(\log x)(\log \log x)})$, with $C$ a suitable positive constant to be chosen later. After some easy considerations, we obtain

$$
\begin{aligned}
& \sum_{\substack{n>x \\
\left(n, a_{2}\right)=1}} \frac{1}{\ell_{u}(n)} \ll_{u} \exp (-C(1 / 3-\varepsilon) \sqrt{(\log x)(\log \log x)}) \\
& \quad+\exp \left(-\frac{1}{2 C}(1-o(1)) \sqrt{(\log x)(\log \log x)}\right)
\end{aligned}
$$


where $o(1)$ tends to zero from the right as $x$ goes to infinity. Now, choosing $C=1 / \sqrt{2(1 / 3-\varepsilon)}$, we see that

$$
\sum_{\substack{n>x \\\left(n, a_{2}\right)=1}} \frac{1}{\ell_{u}(n)} \ll_{u} \exp \left(-\frac{(1-o(1))(1-\varepsilon)}{\sqrt{6}} \sqrt{(\log x)(\log \log x)}\right),
$$

for every $\varepsilon>0$ and $x$ sufficiently large with respect to $\varepsilon$.

\section{Proof of Weak Version of Theorem 1.3}

Proof. We start inserting equation (3.1) inside our main sums.

$$
\sum_{n \leq x}\left(n, u_{n}\right)^{k}=\sum_{n \leq x} \sum_{d \mid\left(n, u_{n}\right)} J_{k}(d)=\sum_{d \leq x} J_{k}(d) \sum_{\substack{n \leq x \\ d \mid\left(n, u_{n}\right)}} 1=\sum_{\substack{d \leq x \\\left(d, a_{2}\right)=1}} J_{k}(d) \sum_{\substack{n \leq x \\ \ell_{u}(d) \mid n}} 1,
$$

by part (3) of Lemma 3.2. Clearly, the last sum in (4.1) is

$$
\sum_{\substack{d \leq x \\\left(d, a_{2}\right)=1}} J_{k}(d)\left\lfloor\frac{x}{\ell_{u}(d)}\right\rfloor \leq x \sum_{\substack{d \leq x \\\left(d, a_{2}\right)=1}} \frac{J_{k}(d)}{\ell_{u}(d)} \leq x \sum_{\substack{d \leq x \\\left(d, a_{2}\right)=1}} \frac{d^{k}}{\ell_{u}(d)}
$$

But now we observe that

$$
\begin{gathered}
\sum_{\substack{d \leq x \\
\left(d, a_{2}\right)=1}} \frac{d^{k}}{\ell_{u}(d)}=\sum_{\substack{d \leq x^{\delta} \\
\left(d, a_{2}\right)=1}} \frac{d^{k}}{\ell_{u}(d)}+\sum_{\substack{x^{\delta}<d \leq x \\
\left(d, a_{2}\right)=1}} \frac{d^{k}}{\ell_{u}(d)} \\
\ll x^{k \delta}+x^{k} \sum_{\substack{d>x^{\delta} \\
\left(d, a_{2}\right)=1}} \frac{1}{\ell_{u}(d)} \\
\ll x^{k} \exp (-(1 / \sqrt{6}-\varepsilon+o(1)) \sqrt{\delta} \sqrt{(\log x)(\log \log x)}),
\end{gathered}
$$

for any $\delta \in(0,1)$, using that the series $\sum_{n} 1 / \ell_{u}(n)$ converges and the bound (1.4), and for any $x$ large in terms of $\delta$ and $\varepsilon$. Now, choosing $\delta$ close to 1 as a function of $\varepsilon$, and by the arbitrarity of $\varepsilon$, we find

$$
\sum_{\substack{d \leq x \\\left(d, a_{2}\right)=1}} \frac{d^{k}}{\ell_{u}(d)} \leq x^{k} \exp (-(1 / \sqrt{6}-\varepsilon+o(1)) \sqrt{(\log x)(\log \log x)}),
$$

where the $o(1)$ depends on $u$ and $k$ and $x$ is chosen large enough with respect to $\varepsilon$. Inserting (4.3) in (4.2) and (4.2) in (4.1), the proof is finished.

\section{Proof of Theorem 1.3}

Proof. Let $y:=\exp \left(\frac{1}{2} \sqrt{(\log x)(\log \log x)}\right)$. We define a partition of $\{n: n \leq x\}$, by setting

$$
\begin{aligned}
& E_{1}(x)=\left\{n \leq x: P(n) \nmid u_{n}\right\} ; \\
& E_{2}(x)=\{n \leq x: P(n) \leq y\} ; \\
& E_{3}(x)=\left\{n \leq x: P(n)>y^{6}, P(n) \in Q_{u, 1 / 3}(x)\right\} ; \\
& E_{4}(x)=\left\{n \leq x: P(n)>y^{6}, P(n) \notin Q_{u, 1 / 3}(x)\right\} ; \\
& E_{5}(x)=\{n \leq x\} \backslash E_{1} \cup E_{2} \cup E_{3} \cup E_{4} .
\end{aligned}
$$

Let $S_{i}=\sum_{n \in E_{i}(x)}\left(n, u_{n}\right)^{k}$, for every $i=\{1,2,3,4,5\}$. We note that if $n \in E_{1}(x)$, then $\left(n, u_{n}\right) \mid(n / P(n))$ and we deduce that

$$
S_{1} \leq \sum_{n \leq x}\left(\frac{n}{P(n)}\right)^{k} \leq x^{k} \sum_{n \leq x} \frac{1}{P(n)^{k}} \leq x^{k+1} \exp ((-\sqrt{2 k}+o(1)) \sqrt{(\log x)(\log \log x)}),
$$


where the last inequality follows by [6, equation 1.6]. Moreover, it is immediate to see that

$$
S_{2} \leq x^{k} \psi(x, y) \leq x^{k+1} \exp (-(1+o(1)) u \log u),
$$

by the Corollary of Theorem 3.1 in [4], where $u=\log x / \log y$ and $o(1)$ tends to zero as $u$ tends to infinity. We observe that we can apply this result because we chose a value of $y$ sufficiently large. Notice also that by our choice of $y$ we have actually got

$$
S_{2} \leq x^{k+1} \exp (-(1+o(1)) \sqrt{(\log x)(\log \log x)}),
$$

which dominates (5.1). Regarding the third sum, we simply use $S_{3} \leq x^{k} \# E_{3}(x)$. Now, if $n \in E_{3}(x)$ we can factorize $n=P(n) m$, with $P(n)>y^{6}$ and $P(n) \in \bar{Q}_{u, 1 / 3}(x)$. This implies that $m<x / y^{6}$ and that $P(n) \in Q_{u, 1 / 3}(x / m)$. Consequently

$$
\# E_{3}(x) \leq \sum_{m \leq x / y^{6}} \# Q_{u, 1 / 3}(x / m) \ll x^{2 / 3} \sum_{m \leq x / y^{6}} \frac{1}{m^{2 / 3}} \ll \frac{x}{y^{2}},
$$

by Lemma 3.3 and a standard final computation. This leads to

$$
S_{3} \ll x^{k+1} \exp (-2 \log y),
$$

which is of the same order of magnitude of (5.2). For what concerns the fourth sum, by part (1) and (6) of Lemma 3.2, we have that $z_{u}(P(n)) \mid n$ and $z_{u}(P(n)) \mid P(n) \pm 1$, implying that $P(n) z_{u}(P(n)) \mid n$. Note that we can affirm the first two divisibility conditions, because we can suppose $P(n) \nmid a_{2} \Delta_{u}$ and odd, since $y$ is large enough. We deduce that

$$
\# E_{4}(x) \leq \sum_{\substack{p>y^{6} \\ p \notin Q_{u, 1 / 3}(x)}} \frac{x}{p z_{u}(p)} \leq \sum_{p>y^{6}} \frac{x}{p^{4 / 3}} \ll \frac{x}{y^{2}}
$$

by a standard computation. Therefore, we find

$$
S_{4} \leq x^{k} \# E_{4}(x) \ll x^{k+1} \exp (-2 \log y),
$$

which coincides with (5.3). We are left then with the estimate of $S_{5}(x)$. To this aim we strictly follow an argument already employed in the proof of [1, Theorem 2]. For any non-negative integer $j$, let $I_{j}:=\left[2^{j}, 2^{j+1}\right)$. We cover $I:=\left[y, y^{6}\right)$ by these dyadic intervals, and we define $a_{j}$ via $2^{j}=y^{a_{j}}$. We shall assume the variable $j$ runs over just those integers with $I_{j}$ not disjoint from $I$. For any integer $k$, define $\mathcal{P}_{j, k}$ as the set of primes $p \in I_{j}$ with $z_{u}(p) \in I_{k}$. Note that, by Lemma 3.3, we have $\# \mathcal{P}_{j, k} \ll 4^{k}$. We have

$$
\begin{gathered}
\# E_{5}(x) \leq \sum_{j} \sum_{k} \sum_{p \in \mathcal{P}_{j, k}} \sum_{\substack{n \leq x \\
P(n) \mid u_{n} \\
P(n)=p}} 1 \leq \sum_{j} \sum_{k} \sum_{p \in \mathcal{P}_{j, k}} \psi\left(\frac{x}{p z_{u}(p)}, p\right) \\
\leq \sum_{j} \sum_{k} \sum_{p \in \mathcal{P}_{j, k}} \frac{x}{p z_{u}(p) y^{2 / a_{j}+o(1)}},
\end{gathered}
$$

as $x \rightarrow \infty$, where we have used the Corollary of Theorem 3.1 in [4] for the last estimate. For $k>j / 2$, we use the estimate

$$
\sum_{p \in \mathcal{P}_{j, k}} \frac{1}{p z_{u}(p)} \leq 2^{-k} \sum_{p \in I_{j}} \frac{1}{p} \leq 2^{-k}
$$

for $x$ large. For $k \leq j / 2$, we use the estimate

$$
\sum_{p \in \mathcal{P}_{j, k}} \frac{1}{p z_{u}(p)} \ll \frac{4^{k}}{2^{j} 2^{k}}=2^{k-j}
$$

since there are at most order of magnitude $4^{k}$ such primes, as noted before. Thus,

$$
\sum_{k} \sum_{p \in \mathcal{P}_{j, k}} \frac{1}{p z_{u}(p)}=\sum_{k>j / 2} \sum_{p \in \mathcal{P}_{j, k}} \frac{1}{p z_{u}(p)}+\sum_{k \leq j / 2} \sum_{p \in \mathcal{P}_{j, k}} \frac{1}{p z_{u}(p)} \ll 2^{-j / 2}=y^{-a_{j} / 2} .
$$


Collecting the above computations, we find

$$
\# E_{5}(x) \leq \sum_{j} \frac{x}{y^{a_{j} / 2+2 / a_{j}+o(1)}}, \text { as } x \rightarrow \infty .
$$

Since the minimum value of $t / 2+2 / t$ for $t>0$ is 2 occuring at $t=2$, we may affirm that

$$
\# E_{5}(x) \leq x / y^{2+o(1)}, \text { as } x \rightarrow \infty,
$$

which leads to an estimate for $S_{5}$ as large as that one for $S_{2}$. We conclude that

$$
\max \left\{S_{1}, S_{2}, S_{3}, S_{4}, S_{5}\right\} \leq x^{k+1} \exp (-(1+o(1)) \sqrt{(\log x)(\log \log x)}),
$$

proving Theorem 1.3.

\section{The multiplicative analogous of $\ell_{u}(n)$}

Let us define the multiplicative function $L_{u}(n)$ such that $L_{u}\left(p^{k}\right)=\ell_{u}\left(p^{k}\right)$, for every prime numbers $p \nmid a_{2}$ and power $k \geq 1$, and $L_{u}\left(p^{k}\right)=p^{k}$, otherwise. Now, consider the Dirichlet series of the function $n / L_{u}(n)$, given by

$$
\alpha(s)=\sum_{n \geq 1} \frac{n}{n^{s} L_{u}(n)} .
$$

Suppose that it converges for $s>\sigma_{c}$, where $\sigma_{c}$ is the abscissa of absolute and ordinary convergence of $\alpha(s)$. Certainly, since $\ell_{u}(n) \leq L_{u}(n)$, for every $n$, and since we know that the series of the reciprocals of $\ell_{u}(n)$ converges, we have $\sigma_{c} \leq 1$. Then, for any $s \in \mathbb{C}$ with $\Re(s)=\sigma>\sigma_{c}$ we can consider the Euler product and it converges to the Dirichlet series in this range. Therefore, we can write

$$
\alpha(s)=\prod_{p \nmid 2 a_{2} \Delta_{u}}\left(1+\sum_{k \geq 1} \frac{f\left(p^{k}\right)}{p^{k s}}\right) \beta(s),
$$

where $f(n)=n / L_{u}(n)$ and $\beta(s)$ is an analytic function in $\Re(s)>0$. Since by property (5) of Lemma 3.2 we find that $f\left(p^{k}\right)=1 / z_{u}(p)$, for any $k \geq 1$ and prime $p \nmid 2 a_{2} \Delta_{u}$, we have

$$
\alpha(s)=\prod_{p \nmid 2 a_{2} \Delta_{u}}\left(1+\frac{f(p)}{p^{s}} \frac{p^{s}}{p^{s}-1}\right) \beta(s)=\prod_{p \nmid 2 a_{2} \Delta_{u}}\left(1+\frac{1}{z_{u}(p)\left(p^{s}-1\right)}\right) \beta(s) .
$$

Now, the final product in (6.1) converges if and only if

$$
\sum_{p \nmid 2 a_{2} \Delta_{u}} \frac{1}{z_{u}(p)\left(p^{s}-1\right)}
$$

converges. Therefore, it suffices to prove that

$$
\lim _{x \rightarrow \infty} \sum_{p>x} \frac{1}{z_{u}(p)\left(p^{\sigma}-1\right)}=0 .
$$

We estimate the last sum separating between primes $p \in \mathscr{Q}_{u, \gamma}$ or $p \notin \mathscr{Q}_{u, \gamma}$. In the first case we obtain

$$
\sum_{\substack{p>x \\ p \in \mathscr{Q}_{u, \gamma}}} \frac{1}{z_{u}(p)\left(p^{\sigma}-1\right)} \ll \int_{x}^{\infty} \frac{d\left(\# \mathscr{Q}_{u, \gamma}(t)\right)}{t^{\sigma}} \ll_{u} \frac{1}{(\sigma-2 \gamma) x^{\sigma-2 \gamma}},
$$

by Lemma 3.3, if we choose $\sigma>2 \gamma$. On the other hand, in the second case we get

$$
\sum_{\substack{p>x \\ p \notin \mathscr{Q}_{u, \gamma}}} \frac{1}{z_{u}(p)\left(p^{\sigma}-1\right)} \ll \sum_{p>x} \frac{1}{p^{\sigma+\gamma}} \ll \frac{1}{(\sigma+\gamma-1) x^{\sigma+\gamma-1}}
$$

if we choose $\sigma+\gamma>1$. Comparing (6.2) with (6.3), we are led to take $\gamma=1 / 3$ and we have showed that

$$
\sum_{p>x} \frac{1}{z_{u}(p)\left(p^{\sigma}-1\right)} \ll_{u} \frac{1}{\varepsilon x^{\varepsilon}}
$$


if $\sigma=2 / 3+\varepsilon$, for every $\varepsilon>0$, and consequently that $\alpha(s)$ converges for every $s$ with $\Re(s)>2 / 3$, or equivalently that $\sigma_{c} \leq 2 / 3$. An immediate application of this result is the following. Let us define

$$
F(s)=\sum_{n \geq 1} \frac{1}{n^{s} L_{u}(n)} .
$$

Then, $F(s)$ has the abscissa of convergence $\sigma_{c}^{\prime} \leq-1 / 3$. This is equivalent to have obtained a strong bound on the tail of $F(0)$. The intermediate passage is made explicit in the next lemma (see e.g. [3, §11.3, Lemma 1]).

Lemma 6.1. Suppose that $G(s)=\sum_{n \geq 1} a_{n} n^{-s}$ is a Dirichlet series of a sequence $\left(a_{n}\right)_{n \geq 1}$ of positive real numbers, with abscissa of convergence $\sigma_{c}^{\prime}$. Suppose that $G(0)$ converges. Then, we have $\sigma_{c}^{\prime}=\inf \left\{\theta: \sum_{n>x} a_{n} \ll x^{\theta}\right\}$.

Since $F(s)$ satisfies the hypotheses of the Lemma 6.1, by (6.4), we deduce that

$$
\sum_{n>x} \frac{1}{L_{u}(n)} \ll_{u} x^{-1 / 3+\varepsilon}
$$

for every $\varepsilon>0$, proving Proposition 1.6.

Remark 6.1. We believe that a finer study of $L_{u}(n)$ could lead to understand better the structure of $\ell_{u}(n)$, though the lack of multiplicativity of the latter makes difficult its study starting with information from the former. For instance, it can be shown that the integers $n$, which have at least two prime factors $p_{1}, p_{2}$ such that a fixed prime $q$ divides both $z_{u}\left(p_{1}\right)$ and $z_{u}\left(p_{2}\right)$, have asymptotic density 1 . Thus, when calculating $z_{u}(n)$ as a least common multiple, there is a cancellation of a factor $q$. In other words, for any positive real number $C$, most integers $n$ have $L_{u}(n) / \ell_{u}(n)>C$. This suggests that the two mentioned functions are not always very close each other.

\section{ACKNOWLEDGEMENTS}

I would like to thank Carlo Sanna for suggesting this problem and for introducing me to the theory of linear recurrences. A special thanks goes also to the anonymous referee, for careful reading and useful advice.

\section{REFERENCES}

1. J. J. Alba González, F. Luca, C. Pomerance, and I. E. Shparlinski, On numbers $n$ dividing the $n$th term of a linear recurrence, Proc. Edinb. Math. Soc. (2) 55 (2012), no. 2, 271-289.

2. R. André-Jeannin, Divisibility of generalized Fibonacci and Lucas numbers by their subscripts, Fibonacci Quart. 29 (1991), no. 4, 364-366.

3. T. Apostol, Introduction to Analytic Number Theory, Springer-Verlag, 1976.

4. E. R. Canfield, P. Erdős and C. Pomerance, On a problem of Oppenheim concerning "Factorisatio Numerorum", J. Number Theory 17 No. 1, 1-28 (1983).

5. G. Everest, A. van der Poorten, I. Shparlinski, T. Ward, Recurrence Sequences, Mathematical Surveys and Monographs, vol.104, American Mathematical Society, Providence, RI, 2003.

6. A. Ivić and C. Pomerance, Estimate for certain sums involving the largest prime factor of an integer, Topics in classical number theory (Budapest, 1981), 769-789, North Holland, 1984.

7. P. Leonetti and C. Sanna, On the greatest common divisor of $n$ and the $n$th Fibonacci number, Rocky Mountain J. Math. (2018).

8. F. Luca and E. Tron, The distribution of self-Fibonacci divisors, Advances in the theory of numbers, Fields Inst. Commun., vol. 77, Fields Inst. Res. Math. Sci., Toronto, ON, 2015, pp. 149-158.

9. M. Renault, The period, rank, and order of the (a, b)-Fibonacci sequence mod m, Math. Mag. 86 (5) (2013) 372-380.

10. C. Sanna, The moments of the logarithm of a G.C.D. related to Lucas sequences, J. of Number Theory (2018).

11. C. Sanna, On numbers $n$ dividing the $n$th term of a Lucas sequence, Int. J. Number Theory 13 (2017), no. 3, $725-734$.

12. C. Sanna, On numbers $n$ relatively prime to the $n$th term of a linear recurrence, Bull. Malays. Math. Sci. Soc.

13. C. Sanna, E. Tron, The density of numbers $n$ having a prescribed G.C.D. with the nth Fibonacci number, Indagationes Mathematicae (2018). 
14. C. Smyth, The terms in Lucas sequences divisible by their indices, J. Integer Seq. 13 (2010), no. 2, Article 10.2.4, 18 .

15. L. Somer, Divisibility of terms in Lucas sequences by their subscripts, Applications of Fibonacci numbers, Vol. 5 (St. Andrews, 1992), Kluwer Acad. Publ., Dordrecht, 1993, pp. 515-525.

16. G. Tenenbaum, Introduction to Analytic and Probabilistic Number Theory, Cambridge U. P., 1995.

Mathematics Institute, Zeeman Building, University of Warwick, Coventry, CV4 7 AL, UK

E-mail address: Daniele.Mastrostefano@warwick.ac.uk 